\title{
Commentary: Identification of potential therapeutic compounds for Parkinson's disease using Drosophila and human cell models
}

\author{
Francisco José Sanz ${ }^{1,2}$, Cristina Solana-Manrique ${ }^{1,2}$, Verónica Muñoz-Soriano ${ }^{1,2}$ and Nuria Paricio ${ }^{1,2 *}$ \\ 'Departamento de Genética, Facultad CC Biológicas, Universidad de Valencia, 46100 Burjassot, Spain \\ ${ }^{2}$ Estructura de Recerca Interdisciplinar en Biotecnologia i Biomedicina (ERI BIOTECMED), Universidad de Valencia, 46100 Burjassot, Spain
}

Article Info

\section{Article Notes}

Received: July 31, 2017

Accepted: September 20, 2017

\section{${ }^{*}$ Correspondence:}

Nuria Paricio

Departamento de Genética, Facultad de CC Biológicas,

Universidad de Valencia

Dr. Moliner 50

E-46100 Burjassot, Spain

Phone: +34-96 3543005

Fax:+34-96 354 3029, Email: nuria.paricio@uv.es

() 2017 Paricio N. This article is distributed under the terms of the Creative Commons Attribution 4.0 International License
Parkinson's disease (PD) is the most common neurodegenerative movement disorder, affecting more than $1 \%$ of 55 -year-old people and more than $3 \%$ of those older than 75 . The best recognized pathological hallmark of PD is the loss of dopaminergic (DA) neurons in the substantia nigra, which leads to a decrease in dopamine levels in the striatum and causes movement impairment ${ }^{1}$. Despite most PD cases are sporadic, several genes responsible for heritable forms of the disease have been described ${ }^{2,3}$. Approximately $5-10 \%$ of patients suffer for a monogenic form of PD where dominant mutations in SNCA, LRRK2 and VP35 or recessive mutations in PINK, DJ-1 and Parkin cause the disease with high penetrance ${ }^{2}$. Functional studies of these genes have been essential to identify the mechanisms underlying the neuropathology of $\mathrm{PD}^{3}$. Interestingly, genome-wide association studies confirm that some of these genes are also affected in sporadic PD forms ${ }^{3}$.

Mutations in recessive PD genes like PINK, DJ-1 and Parkin are relatively rare in the general $\mathrm{PD}$ population, but they are responsible of most cases of juvenile PD (mean onset age of $\sim 39$ years) ${ }^{2}$. It has been shown that they regulate common pathways, being involved in mitochondrial quality control, protein degradation processes and oxidative stress response ${ }^{4}$. In our group, we are interested in $D J-1$, which was first identified as a causative PD gene in consanguineous Dutch and Italian families that displayed early-onset $\mathrm{PD}^{5}$. Although it was initially described as an oncogene, subsequent studies have shown that it is also involved in mitochondrial homeostasis and defense against oxidative stress $(\mathrm{OS})^{6}$. The DJ-1 protein is localized in the nucleus and the cytoplasm, but it is also present in mitochondria under OS conditions. Therefore, it has been proposed that DJ-1 is able to perform different functions depending on its subcellular localization? ${ }^{7}$.

At present, PD is an incurable disease for which existing therapies are not sufficiently effective to stop or delay its progression. They are mainly based on a dopamine replacement strategy, capable of alleviating PD motor symptoms ${ }^{8}$. Common characteristics have been recently described in several neurodegenerative disorders such as increase in inflammation and OS levels, protein aggregation and ion homeostasis alterations ${ }^{9}$. For this reason, compounds with antioxidant, anti-inflammatory and neuroprotective activities are currently being considered as potential therapies for those disorders, including PD. Animal models of human diseases are commonly used in elucidating the biological pathways and environmental factors that influence their clinical presentation. In addition, they can be used in compound and small-molecule screening for the development of new therapeutic 
strategies. In such a scenario, studies in Drosophila models have acquired a prominent position in providing insight into human biology and disease. Despite the apparent differences that do exist between Drosophila and humans, biological pathways and essential cellular processes are very similar in both organisms. This, together with its experimental tractability, makes Drosophila a very useful tool to identify new therapeutic compounds for human diseases ${ }^{10,11}$. In addition, chemical screens in a living animal such as Drosophila avoid common problems of traditional in vitro screens, in which lead compounds identified often fail to exert the expected effect in murine models due to suboptimal pharmacokinetics and pharmacodynamics ${ }^{12}$.

For several years, we have been working with a Drosophila model of familial PD based on inactivation of the $D J-1 \beta$ gene (ortholog of human $D J-1$ ). Previous studies from our group demonstrated that $D J-1 \beta$ mutant flies exhibit motor defects, shortened lifespan, increased levels of reactive oxygen species (ROS) and other OS markers, and hypersensitivity to OS-inducing toxins ${ }^{13-15}$. Moreover, we also showed that some of these phenotypes could be suppressed with antioxidant treatments ${ }^{15}$. In an attempt to identify new potential drugs to treat PD, we recently analyzed the ability of several antioxidant, anti-inflammatory and neuroprotective compounds to suppress motor deficits of $D J-1 \beta$ mutants using a climbing assay ${ }^{16}$. This assay is a useful and easy method to evaluate locomotor ability in Drosophila, and is based on the negative geotactic behavior shown by this organism. It has been widely used to test the effectiveness of compounds to suppress motor deficits in Drosophila models of several neurodegenerative diseases, including familial PD based on mutations in $S N C A^{17-21}, L R R K 2^{22-24}$, PINK1 $1^{25,26}$ or parkin ${ }^{27-30}$. In our study, we selected 23 compounds already tested in humans and whose function or activity could be beneficial in a context of $D J-1 \beta$ deficiency. Additionally, their mechanisms of action were different from those of existing therapies used for PD treatment, and some of them were shown to be well-suited for other medical applications. Then, we performed a chemical screen to test the efficacy of those drugs to specifically suppress locomotor defects in $D J-1 \beta$ mutants. For doing so, model and control flies were cultured in medium supplemented with each compound during development plus five days after eclosion, and then were subjected to climbing assays. We found that some of them were beneficial for both control and $D J-1 \beta$ mutant flies, like celastrol, methylcobalamine, piracetam, metformin and acetylsalicylic acid, and others only for control flies, like curcumin. However, seven compounds were able to improve motor ability specifically in $D J-1 \beta$ mutant flies (dexrazoxane, pterostilbene, tocopherol, sodium phenylbutyrate, dalfampridine, methylene blue and minocycline), which were selected for subsequent analysis. At this point, we wondered how these compounds could be exerting their beneficial effect in $D J-1 \beta$ mutants, and focused on their possible impact in ROS production and protein oxidative damage. Both human DJ-1 and DJ-1ß proteins protect against oxidative insults and are modified by oxidation ${ }^{6,31}$. Consistently, $D J-1 \beta$ mutant flies displayed high levels of $\mathrm{H}_{2} \mathrm{O}_{2}$ (a component of the ROS pool) and of carbonylated proteins, when compared to control flies ${ }^{14,15}$. Our results showed that while three of the seven candidate compounds were capable of reducing $\mathrm{H}_{2} \mathrm{O}_{2}$ levels (tocopherol, dalfampridine and minocycline), all of them but sodium phenylbutyrate also decreased the amount of carbonylated proteins. This is a very interesting finding because it suggests that this particular type of protein modification has a causative role in the locomotor defects of $D J-1 \beta$ mutants. Carbonyl groups are added to proteins when ROS levels are elevated, but also through metal-catalyzed oxidation ${ }^{32}$. In addition, non-oxidative mechanisms can also effectively increase carbonylation levels in proteins ${ }^{33}$. Protein carbonylation is an irreversible posttranslational modification that generally leads to protein inactivation and degradation, therefore affecting cellular functions ${ }^{34}$. In such a scenario, our current interest is to identify the specific proteins affected by this damage, and probably inactivated, in PD model flies. This will help to further determine cellular processes underlying $D J-1 \beta$ inactivation and DJ-1-linked PD pathogenesis. Together, the results obtained in the Drosophila PD model indicate that the molecular mechanism by which the candidate compounds exert their beneficial effect are diverse, thus confirming the complexity of PD pathogenesis ${ }^{8}$.

Many studies performed in Drosophila models of human diseases have demonstrated that this organism can contribute to clinically relevant translational research in drug discovery ${ }^{12}$. Therefore, to evaluate the translatability of the results obtained in flies we used undifferentiated human neuroblastoma SH-SY5Y cells, an in vitro model widely used in PD research ${ }^{35}$. Several toxin-induced as well as genetic PD models have been obtained in these cells and used to test the therapeutic potential of compounds identified in other organisms ${ }^{24,36-38}$. In our study, we generated DJ-1-deficient SH-SY5Y cells, in which the DJ-1 gene was silenced by $\mathrm{RNAi}^{16}$. Indeed, DJ-1 protein levels were reduced up to $90 \%$ in such cells. MTT assays showed that DJ-1-deficient cells were more susceptible to $\mathrm{H}_{2} \mathrm{O}_{2}$ induced dose-dependent cytotoxicity than control cells, consistent with previous studies ${ }^{39}$. Interestingly, we found that pretreatment of $D J-1$ mutant cells with all candidate compounds butpterostilbene was able to reduce OS-induced cytotoxicity, thus supporting the therapeutic potential of the compounds identified in the Drosophila PD model. Previous studies have demonstrated that pterostilbene exerts its neuroprotective function by inhibiting microglial activation $^{40}$; hence, this compound is probably beneficial in $D J-1 \beta$ mutant flies (a whole animal model) through 
microglial-like cells inactivation in the nervous system, despite being unable to suppress OS-induced cytotoxicity in DJ-1-deficient SH-SY5Y cells. In consequence, our study demonstrates that there is a good correlation between the results obtained in the Drosophila model and in DJ1-deficient human cells, thus confirming the use of this organism in drug discovery for human diseases. It should be mentioned that all the candidate compounds identified in this work have been already tested in humans, and some of them have been used to treat patients with PD and other neurodegenerative disorders with different results. While some were able to alleviate symptoms in PD patients, others failed to provide improved clinical outcomes ${ }^{41,42}$. Based on these data and considering the different mechanisms of action of the compounds identified, the development of a combinatorial therapy approach for PD targeting different cellular processes (not only OS) may result in additive or synergistic relief of pathology ${ }^{41}$.

Although Drosophila is not a substitute for preclinical testing in mammals, our study demonstrates that it can be an important component in drug discovery workflows when harnessed for efficient and high-throughput screenings ${ }^{43}$. It also shows that the climbing assay is an easy method to identify compounds able to improve locomotor abilities in fly models of neurological disorders with movement impairments. This is especially relevant in the search of new effective therapies for PD because current treatments do not stop or delay the progression of the disease, and present problems in chronic treatments. As mentioned above, most PD cases are sporadic; however, several genes involved in rare familial forms of the disease appear to be affected in sporadic $\mathrm{PD}^{3}$. It has been shown that causative genes to PD like parkin or LRRK2 are misregulated in sporadic PD cases $^{44,45}$. Regarding the $D J-1$ gene, previous studies have demonstrated that the DJ-1 protein is oxidatively modified, and probably inactivated, in sporadic PD cases $^{46}$. Besides, it has been recently suggested that $\alpha$-Synuclein aggregates, which are the main components of Lewy bodies present in sporadic forms of $\mathrm{PD}$, may recruit DJ-1 to form insoluble inclusions, thereby attenuating its antioxidant neuroprotective ability $\left(\mathrm{see}^{47}\right)$. Therefore, the results obtained in the Drosophila model of familial PD based on $D J-1 \beta$ inactivation, and validated in $D J$ 1-deficient human neuroblastoma cells, might be relevant for sporadic PD forms.

In conclusion, we hypothesize that any compound showing a rescue effect both in Drosophila and human cell PD models based on $D J-1$ deficiency would be likely to exert a beneficial effect in future clinical trials. However, further analyses will be required to confirm this assumption. Besides, we believe that the potential therapeutic compounds identified in our study could be useful to treat not only PD patients with mutations in the $D J-1$ gene but also those affected by sporadic PD. The next step will be to perform high-throughput chemical screens using $D J$ $1 \beta$ mutant flies in an attempt to discover alternative and hopefully more effective PD treatments.

\section{Acknowledgements}

This work was supported by grants from Generalitat Valenciana (PROMETEO/2010/08 and PROMETEO/2014/067).

\section{References}

1. Farlow J, Pankratz ND, Wojcieszek J, et al. Parkinson disease overview. GeneReviews. 2014; Internet: 1993-2016.

2. Lill CM. Genetics of Parkinson's disease. Mol Cell Probes. 2016; 30(6): 386-396. doi:10.1016/j.mcp.2016.11.001.

3. Poewe W, Seppi K, Tanner CM, et al. Parkinson disease. Nat Rev Dis Prim. 2017; 3: 17013. doi:10.1038/nrdp.2017.13.

4. Scott L, Dawson VL, Dawson TM. Trumping neurodegeneration: Targeting common pathways regulated by autosomal recessive Parkinson's disease genes. Exp Neurol. 2017; S0014-4886(17): 30102-30104. doi:10.1016/j.expneurol.2017.04.008.

5. Bonifati V, Rizzu P, van Baren MJ, et al. Mutations in the DJ-1 gene associated with autosomal recessive early-onset parkinsonism. Science (80-) ). 2003; 299: 256-259.

6. Ariga H, Takahashi-Niki K, Kato I, et al. Neuroprotective function of DJ-1 in Parkinson's disease. Oxid Med Cell Longev. 2013; 2013: 1-9.

7. van der Merwe C, Jalali Sefid Dashti Z, Christoffels A, et al. Evidence for a common biological pathway linking three Parkinson's disease-causing genes: Parkin, PINK1 and DJ-1. Eur J Neurosci. 2015; 41: 1113-1125.

8. Athauda D, Foltynie T. The ongoing pursuit of neuroprotective therapies in Parkinson disease. Nat Rev Neurol. 2015; 11: 25-40.

9. Rasool M, Malik A, Qureshi MS, et al. Recent updates in the treatment of neurodegenerative disorders using natural compounds. Evidencebased Complement Altern Med. 2014; 2014: 1-7.

10. Lawal HO, Terrell A, Lam HA, et al. Drosophila modifier screens to identify novel neuropsychiatric drugs including aminergic agents for the possible treatment of Parkinson's disease and depression. Mol Psychiatry. 2014; 19(2): 235-242.

11. Perrimon N, Bonini NM, Dhillon P. Fruit flies on the front line: the translational impact of Drosophila. Dis Model Mech. 2016; 9: 229-231.

12. Fernández-Hernández I, Scheenaard E, Pollarolo G, et al. The translational relevance of Drosophila in drug discovery. EMBO Rep. 2016; 17(4): 471-472. doi:10.15252/embr.201642080.

13. Lavara-Culebras E, Paricio N. Drosophila DJ-1 mutants are sensitive to oxidative stress and show reduced lifespan and motor deficits. Gene. 2007; 400: 158-165.

14. Lavara-Culebras E, Muñoz-Soriano V, Gómez-Pastor R, et al. Effects of pharmacological agents on the lifespan phenotype of Drosophila DJ1beta mutants. Gene. 2010; 462: 26-33.

15. Casani S, Gómez-Pastor R, Matallana E, et al. Antioxidant compound supplementation prevents oxidative damage in a Drosophila model of Parkinson's disease. Free Radic Biol Med. 2013; 61: 151-160.

16. Sanz FJ, Solana-manrique C, Muñoz-soriano V, et al. Identification of potential therapeutic compounds for Parkinson's disease using Drosophila and human cell models. Free Radic Biol Med. 2017; 108: 683-691.

17. Siddique $\mathrm{YH}$, Ara $\mathrm{G}$, Jyoti $\mathrm{S}$, et al. The dietary supplementation of nordihydroguaiaretic acid (NDGA) delayed the loss of climbing ability 
in Drosophila model of Parkinson's disease. J Diet Suppl. 2012; 9(1): 1-8. doi:10.3109/19390211.2011.630716.

18. Siddique $\mathrm{YH}$, Jyoti S, Naz F. Effect of epicatechin gallate dietary supplementation on transgenic Drosophila model of Parkinson's disease. J Diet Suppl. 2014; 11(2): 121-130. doi:10.3109/19390211. 2013.859207.

19. Siddique YH, Naz F, Jyoti S, et al. Protective effect of Geraniol on the transgenic Drosophila model of Parkinson's disease. Environ Toxicol Pharmacol. 2016; 43: 225-231.

20. Liu LF, Song JX, Lu JH, et al. Tianma Gouteng Yin, a Traditional Chinese Medicine decoction, exerts neuroprotective effects in animal and cellular models of Parkinson's disease. Sci Rep. 2015; 5: 16862. doi:10.1038/srep16862.

21. Styczyńska-Soczka K, Zechini L, Zografos L. Validating the Predicted Effect of Astemizole and Ketoconazole Using a Drosophila Model of Parkinson's Disease. Assay Drug Dev Technol. 2017; 15(3): 106-112. doi:10.1089/adt.2017.776.

22. Angeles DC, Ho P, Dymock BW, et al. Antioxidants inhibit neuronal toxicity in Parkinson's disease-linked LRRK2. Ann Clin Transl Neurol. 2016; 3(4): 288-294. doi:10.1002/acn3.282.

23. De Rose F, Marotta R, Poddighe S, et al. Functional and Morphological Correlates in the Drosophila LRRK2 loss-of-function Model of Parkinson's Disease: Drug Effects of Withania somnifera (Dunal) Administration. PLoS One. 2016; 11(1): e0146140. doi:10.1371/ journal.pone.0146140.

24. Lin CH, Lin $\mathrm{HI}$, Chen ML, et al. Lovastatin protects neurite degeneration in LRRK2-G2019S parkinsonism through activating the Akt/Nrf pathway and inhibiting GSK3beta activity. Hum Mol Genet. 2016; 25(10): 1965-1978.

25. Poddighe S, De Rose F, Marotta R, et al. Mucuna pruriens (Velvet bean) rescues motor, olfactory, mitochondrial and synaptic impairment in PINK1B9 Drosophila melanogaster genetic model of Parkinson's disease. PLoS One. 2014; 9(10): e110802. doi:10.1371/journal. pone. 0110802 .

26. Jansen RLM, Brogan B, Whitworth AJ, et al. Effects of five Ayurvedic herbs on locomotor behaviour in a Drosophila melanogaster Parkinson's disease model. Phyther Res. 2014; 28(12): 1789-1795. doi:10.1002/ptr.5199.

27. Bonilla-Ramirez L, Jimenez-Del-Rio M, Velez-Pardo C. Low doses of paraquat and polyphenols prolong life span and locomotor activity in knock-down parkin Drosophila melanogaster exposed to oxidative stress stimuli: implication in autosomal recessive juvenile parkinsonism. Gene. 2013; 512(2): 355-363. doi:10.1016/j. gene.2012.09.120

28. Chambers RP, Call GB, Meyer D, et al. Nicotine increases lifespan and rescues olfactory and motor deficits in a Drosophila model of Parkinson's disease. Behav Brain Res. 2013; 253: 95-102. doi:10.1016/j.bbr.2013.07.020.

29. Srivastav S, Singh SK, Yadav AK, Srikrishna S. Folic acid supplementation rescues anomalies associated with knockdown of parkin in dopaminergic and serotonergic neurons in Drosophila model of Parkinson's disease. Biochem Biophys Res Commun. 2015; 460(3): 780-785.

30. Srivastav S, Singh SK, Yadav AK, et al. Folic Acid Supplementation Ameliorates Oxidative Stress, Metabolic Functions and Developmental Anomalies in a Novel Fly Model of Parkinson's Disease. Neurochem Res. 2015; 40(7): 1350-1359. doi:10.1007/s11064-015-1598-x.
31. Meulener MC, Xu K, Thomson L, et al. Mutational analysis of DJ-1 in Drosophila implicates functional inactivation by oxidative damage and aging. Proc Natl Acad Sci U S A. 2006; 103: 12517-12522.

32. Møller IM, Rogowska-Wrzesinska A, Rao RSP. Protein carbonylation and metal-catalyzed protein oxidation in a cellular perspective. $J$ Proteomics. 2011; 74: 2228-2242.

33. Adams S, Green $\mathrm{P}$, Claxton $\mathrm{R}$, et al. Reactive carbonyl formation by oxidative and non-oxidative pathways. Front Biosci. 2001; 6: A17-24.

34. Grimsrud PA, Xie H, Griffin TJ, et al. Oxidative stress and covalent modification of protein with bioactive aldehydes. J Biol Chem. 2008; 283(32): 21837-21841.

35. Xicoy H, Wieringa B, Martens GJM. The SH-SY5Y cell line in Parkinson's disease research: a systematic review. Mol Neurodegener. 2017; 12 : $1-11$.

36. Li X, Zhang S, Wang $\mathrm{K}$, et al. Neuroprotective effects of extract of Acanthopanax senticosus harms on SH-SY5Y cells overexpressing wild-type or A53T mutant $\alpha$-synuclein. Phytomedicine. 2014; 21(5) 704-711. doi:10.1016/j.phymed.2013.10.012.

37. van der Merwe C, van Dyk HC, Engelbrecht L, et al. Curcumin Rescues a PINK1 Knock Down SH-SY5Y Cellular Model of Parkinson's Disease from Mitochondrial Dysfunction and Cell Death. Mol Neurobiol. 2017; 54(4): 2752-2762. doi:10.1007/s12035-016-9843-0.

38. Yang J, Kim MJ, Yoon W, et al. Isocitrate protects DJ-1 null dopaminergic cells from oxidative stress through NADP+-dependent isocitrate dehydrogenase (IDH). PLoS Genet. 2017; 13(8): e1006975. doi:10.1371/journal.pgen.1006975.

39. Wang Z, Liu J, Chen S, et al. DJ-1 modulates the expression of $\mathrm{Cu}$ / Zn-superoxide dismutase-1 through the Erk1/2-Elk1 pathway in neuroprotection. Ann Neurol. 2011; 70: 591-600.

40. Hou Y, Xie G, Miao F, et al. Pterostilbene attenuates lipopolysaccharide-induced learning and memory impairment possibly via inhibiting microglia activation and protecting neuronal injury in mice. Prog Neuro-Psychopharmacology Biol Psychiatry. 2014; 54: 92-102.

41. Olson KE, Gendelman HE. Immunomodulation as a neuroprotective and therapeutic strategy for Parkinson's disease. Curr Opin Pharmacol. 2016; 26: 87-95

42. The Parkinson Study Group. Effects of tocopherol and deprenyl on the progression of disability in early Parkinson's disease. $N$ Engl J Med. 1993; 328(3): 176-183.

43. Martin I, Chittoor VG. Parkinson disease: Insect screens for PD therapies - keep the flies in. Nat Rev Neurol. 2016; 12: 318-319.

44. Chai C, Lim KL. Genetic insights into sporadic Parkinson's disease pathogenesis. Curr Genomics. 2013; 14(8): 486-501. doi:10.2174/138 9202914666131210195808.

45. Lesage S, Brice A. Role of mendelian genes in "sporadic" Parkinson's disease. Parkinsonism Relat Disord. 2012; 18 Suppl 1: 66-70. doi:10.1016/S1353-8020(11)70022-0.

46. Jiang T, Sun Q Chen S. Oxidative stress: A major pathogenesis and potential therapeutic target of antioxidative agents in Parkinson's disease and Alzheimer's disease. Prog Neurobiol. 2016; 147: 1-19.

47. Kim T, Vemuganti R. Mechanisms of Parkinson's disease-related proteins in mediating secondary brain damage after cerebral ischemia. J Cereb blood flow Metab. 2017; 37(6): 1910-1926. doi:10.1177/0271678X17694186. 